\title{
LEVANTAMENTO DAS UNIDADES DE CONSERVAÇÃO MARINHAS E COSTEIRAS FEDERAIS BRASILEIRAS
}

\author{
ROCHA, M. M. ${ }^{1 *}$; MARENZI, R. C. ${ }^{1}$ JUNIOR, I. F. L. ${ }^{1}$ \& AZEVEDO, M. ${ }^{1}$ \\ 1. Laboratório de Conservação e Gestão Costeira da Universidade do Vale do Itajaí - UNIVALI. \\ *Autor correspondente: matheusmrochasc@gmail.com
}

\begin{abstract}
Rocha, M. M., Marenzi, R. C., Junior, I. F. L. \& Azevedo, M. (2020). Survey of Brazilian Coastal and Marine Protected. Braz. J. Aquat. Sci. Technol. 24(2). eISSN 1983-9057. DOI: 15928/bjast.v24n2. One of the most efficient ways to protect ecosystems and biodiversity is the creation of protected areas (PA). This paper aims to analyze the Brazilian Coastal and Marine Protected Areas. Through the National Register of Protected Areas, the Federal APs were downloaded and those existing in the marine ecosystem or located from the shoreline to the limit of $50 \mathrm{~km}$ to the continent were selected. 74 UCs were found, of which 33 were in full use, where Monte Pascoal National Park was the first protected UC created in the coastal ecosystem in 1961, just 42 years after the creation of the first Brazilian conservation unit, Itatiaia National Park. The others (41) are from the Sustainable Use group. Of these, the first containing marine and coastal ecosystems were the Piaçabuçú and Cairuçu Environmental Protection Areas (EPA), both created in 1993. Therefore, 11 years after the creation of the first sustainable use PAs of the Petrópolis Mountainous Region and EPA Paraíba do Sul River Basin, in 1982. The survey showed that only 43 (48\%) of the PAs have a management plan and 5 are still under construction. On the other hand, in 2018 there was a substantial increase in PAareas, especially considering the Trindade and Martim Vazand São Pedro and São PauloArchipelago EPAs, and their Natural Monuments of the same name, from $1.6 \%$ to approximately $25 \%$ of the country's legally protected waters. Exceeding the goals of Aichi and SDG 14, but most importantly, the quality of management in order to meet the objectives of each PA, which result in the effective maintenance of biodiversity and natural and cultural resources.
\end{abstract}

Key Words: Protected Areas, Coastal Management, Goal 14.

\section{INTRODUÇÃO}

Do contato com a natureza para a sobrevivência humana, as áreas naturais passaram a ter um significado especial desde a cultura pré-agrária na Ásia, em que civilizações antigas já mantinham áreas protegidas (Terborgh, 2002). Nessa época, as áreas naturais eram associadas à presença de animais sagrados, de plantas medicinais, de água pura, de matéria-prima futura ou aos mitos e ocorrências históricas (Marenzietal., 2013). Outras ações relativas conservação da natureza foram sendo realizadas, até que em 1892 houve a criação do primeiro parque nacional do mundo, o Yellowstone National Park, nos Estados Unidos. Este foi um marco para que outros países estabelecessem as suas primeiras áreas legalmente protegidas.

No Brasil, as primeiras iniciativas voltadas à proteção de áreas têm seu registro ainda no período colonial, em que o principal objetivo era a garantia do controle sobre o manejo de determinados recursos, como a madeira ou a água, conforme práticas em algumas partes da Europa (Medeiros, 2006). Atualmente há diferentes modalidades de áreas protegidas, que são reconhecidas pela legislação ambiental, como as Áreas de Preservação Permanente - APP, as Reservas Legais e as Unidades de Conservação. Contudo, as UCs têm ato legal de criação próprio e limites e objetivos definidos, diferenciando-as das demais áreas protegidas (Brasil, 2000).
A primeira unidade de conservação brasileira foi criada em 1937, o Parque Nacional do Itatiaia, mas já em 1876 o Eng. André Rebouças propôs a criação de parques nacionais em Sete Quedas e na Ilha do Bananal, sendo o primeiro decretado em 1959 e o segundo em 1961, como Parque Nacional do Araguaia (Pádua, 2004). Muitas outras UCs foram criadas, mas sem um padrão e critério conceitual, assim como no restante dos países, os quais diferem na interpretação conceitual e administrativa, em que uma área protegida em um país não será necessariamente entendida como tal em outro (IUCN, 2008). No Brasil, a Lei Federal no 9.985 de 18 de julho de 2000 estabeleceu o Sistema Nacional de Unidades de Conservação - SNUC,padronizando conceitos e reconhecendo doze categorias de unidades de conservação, divididas em dois grupos: UCs de Proteção Integral e de Uso Sustentável.

As unidades de conservação de Proteção Integral visam preservar a natureza, sendo permitido apenas o uso indireto dos seus recursos naturais (Brasil, 2000). Caracterizam-se por possuírem ecossistemas que necessitam de maiores cuidados devido sua fragilidade e particularidades (Martins et al., 2015). Já as unidades de Uso Sustentável têm como objetivo compatibilizar a conservação da natureza com o uso sustentável de parte dos seus recursos naturais (Brasil, 2000).

OSNUC é constituído pelo conjunto das unidades de conservação federais, estaduais e municipais 
e entre as suas diretrizes estabelece que no conjunto das unidades de conservação estejam representadas amostras significativas e ecologicamente viáveis dos diferentes ecossistemas do território nacional e das águas jurisdicionais. Contudo, no estudo referente ao panorama da conservação dos ecossistemas costeiros e marinhos no Brasil, o "ecossistema marinho" representa a grande lacuna do sistema, demandando medidas urgentes visando o planejamento de sua conservação (MMA, 2010). Em 2010, o Brasil contava com 1.540.887 hectares de UCs que abrigavam a área costeira e marinha, sendo que destes, 156.264 hectares correspondiam a nove UCs exclusivamente marinhas, representando 10\% de área (Drummond, 2010). Esta situação mudou em termos de representação espacial com a criação das UCs dos arquipélagos de São Pedro e São Paulo e Trindade e Martim Vaz, ainda que necessário considerar a efetividade de gestão dessas.

Os ecossistemas marinhos possuem uma série de peculiaridades que resultam emuma maior complexidade à gestão, uma vez que o conhecimento historicamente foi construído com o foco em sistemas terrestres (Soares et al., 2010), inclusive considerando os métodos de ecologia da paisagem tão importantes no planejamento e implantação de UCs. Além disso, as UCs na zona costeira não estão distribuídas uniformemente e poucas são exclusivamente marinhas (Prates \& Pereira, 2000).

O esforço da representatividade de ecossistemas a serem protegidos é também de outros países, sendo usual o termo Área Marinha Protegida (AMP), reconhecido como referente a regiões compostas pelo mar e zonas costeiras, podendo ou não estar diretamente ligadas aos oceanos (Salm et al., 2000). Assim, entre as políticas públicas voltadas as AMPsdestacam-se o ODS 14, que trata da Vida na Água e estabelece um conjunto de metas, entre as quais, já para 2020 prevê conservar pelo menos $10 \%$ das zonas costeiras e marinhas, o que vai de encontro a Convenção da Diversidade Biológica (CDB) das Nações Unidas, em Nagóia, em 2010, conhecida como Meta de Aichi.

Com base na necessidade de atender as políticas públicas ambientais e entender o processo de criação de AMPs ao longo do tempo no Brasil, este trabalho objetivou levantar a situação das Unidades de Conservação Marinhas e Costeiras criadas na esfera Federal.

\section{MATERIAIS E MÉTODOS}

Esta pesquisa tem caráter exploratório, cujos dados foram obtidos do Cadastro Nacional de Unidades de Conservação (CNUC) por meio do site oficial do Ministério do Meio Ambiente (MMA), disponibilizados em formato SHP. Deste, foram baixados os arquivos referentes às Unidades de Conservação federais e as informações geográficas foram processadas com o auxílio de software de geoprocessamento ArcGis.

Apesar de que o ICMBio lista as UCs por Bioma (Amazônia, Cerrado, Caatinga, Pantanal, Pampa, Mata Atlântica, Marinho), nesse trabalho desenvolveu-se classificação própria com fins de atender o objetivo do levantamento. Assim, as UCs levantadas foram classificadas em Marinhas, Marinhas-Costeiras e Costeiras, a partir dos seguintes critérios: 1) UCs Marinhas - localizadas em área territorial fora do continente terrestre, incluindo apenas o mar e as ilhas; 2) UCs Marinhas-Costeiras - localizadas em área continental terrestre com extensão de parte em ecossistema marinho; 3) UCs Costeiras - que constavam no site do ICMBio no Bioma Marinho Costeiro e estavam localizadas da linha da costa até o limite de $50 \mathrm{~km}$ para o continente ou no Bioma Mata Atlântica e porção estava localizada em área de praia.

Para caracterização, após classificação e levantamento, foram pesquisados em sites de instituições reconhecidas os dados complementares sobre ato legal de criação, área, municípios de abrangência, e tipo de ecossistema representado, separadas em UCs de proteção integral e de uso sustentável.

\section{RESULTADOS}

Foram levantadas 74 UCs em ecossistemas marinhos e costeiros, sendo 33 de proteção integral e 41 de uso sustentável. Nas Tabelas 1 e 2 é possível verificar dados do levantamento das UCs Marinhas, Marinhas-Costeiras e Costeiras no âmbito Federal, em ordem cronológica de criação, e demais informações pertinentes.

Das 33 UCs de proteção integral levantadas, $14(42,4 \%)$ são exclusivamente marinhas incluindo as ilhas, $11(33,3 \%)$ costeiras e oito $(24,2 \%)$ marinho costeiras. A Figura 1 demonstra as UCs de proteção integral em termos de área e de quantidade ao longo das décadas.

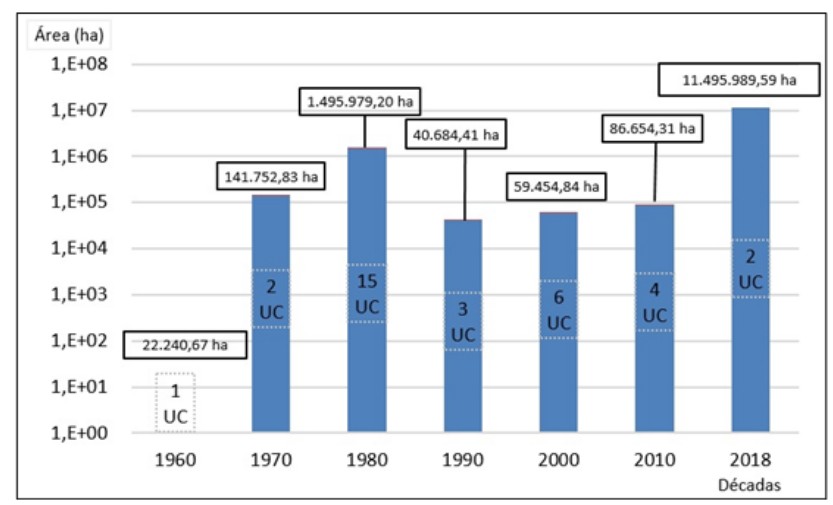

Figura 1 - Representação das Unidades de Conservação de proteção Integral marinhas e/ou costeiras em relação a sua área e quantidade ao longo das décadas. 
Tabela 1 - Unidades de Conservação Marinhas e Costeiras Federais de Proteção Integral.

\begin{tabular}{|c|c|c|c|c|c|}
\hline $\begin{array}{l}\text { Unidades de } \\
\text { conservação } \\
\end{array}$ & Ato legal & Área (ha) & Municípios & Ecossistema & $\begin{array}{c}\text { Plano de } \\
\text { manejo }\end{array}$ \\
\hline $\begin{array}{l}\text { PARNA DO MONTE } \\
\text { PASCOAL* }\end{array}$ & $\begin{array}{l}\text { Decreto } n^{\circ} 242, \text { de } 29 \text { de } \\
\text { novembro de } 1961\end{array}$ & $22.240,67$ & Porto Seguro, Prado - BA & Costeiro & Sim \\
\hline $\begin{array}{l}\text { PARNA DA SERRA } \\
\text { DA BOCAINA* }^{*}\end{array}$ & $\begin{array}{l}\text { Decreto } n^{\circ} 68.172, \text { de } 04 \text { de } \\
\text { fevereiro de } 1971\end{array}$ & $106.566,42$ & $\begin{array}{c}\text { Parati, Angra dos Reis, } \\
\text { Carapebus - RJ } \\
\text { Ubatuba, } \\
\text { Areias, Cunha, São José do } \\
\text { Barreiro - SP }\end{array}$ & Costeiro & Sim \\
\hline $\begin{array}{l}\text { REBIO ATOL DAS } \\
\text { ROCAS }\end{array}$ & $\begin{array}{c}\text { Decreto } n^{\circ} 83.549 \text {, de } 05 \text { de } \\
\text { junho de } 1979\end{array}$ & $35.186,41$ & Natal - RN & Marinho & Sim \\
\hline $\begin{array}{l}\text { PARNA DO CABO } \\
\text { ORANGE }\end{array}$ & $\begin{array}{c}\text { Decreto } n^{\circ} 84.913, \text { de } 15 \text { de } \\
\text { julho de } 1980\end{array}$ & $657.318,06$ & Oiapoque, Calçoene - AP & $\begin{array}{l}\text { Marinho } \\
\text { Costeiro }\end{array}$ & Sim \\
\hline $\begin{array}{l}\text { REBIO DO LAGO } \\
\text { PIRATUBA* }\end{array}$ & $\begin{array}{c}\text { Decreto } n^{\circ} 84.914 \text {, de } 16 \text { de } \\
\text { julho de } 1980\end{array}$ & $392.474,85$ & $\begin{array}{c}\text { Amapá, Tartarugalzinho, } \\
\text { Pracuúba - AP }\end{array}$ & Costeiro & Não \\
\hline REBIO DE UNA* & $\begin{array}{l}\text { Decreto } n^{\circ} 85.463 \text {, de } 10 \text { de } \\
\text { dezembro de } 1980\end{array}$ & $18.715,06$ & Una - BA & Costeiro & Sim \\
\hline $\begin{array}{l}\text { PARNA DOS } \\
\text { LENÇOIS } \\
\text { MARANHENSES }\end{array}$ & $\begin{array}{c}\text { Decreto } n^{\circ} 86.060 \text {, de } 02 \text { de } \\
\text { junho de } 1981\end{array}$ & $156.608,16$ & $\begin{array}{c}\text { Barreirinha, Santo Amaro do } \\
\text { Maranhão, Primeira Cruz, } \\
\text { Barreirinhas - MA }\end{array}$ & $\begin{array}{l}\text { Marinho } \\
\text { Costeiro }\end{array}$ & Sim \\
\hline $\begin{array}{l}\text { ESEC DE MARACÁ } \\
\text { JIPIOCA }\end{array}$ & $\begin{array}{c}\text { Decreto } s / n^{\circ} \text {, de } 02 \text { de junho de } \\
1981\end{array}$ & $58.756,95$ & Amapá - AP & $\begin{array}{l}\text { Marinho } \\
\text { Costeiro }\end{array}$ & Sim \\
\hline $\begin{array}{l}\text { PARNA MARINHO } \\
\text { DOS ABROLHOS }\end{array}$ & $\begin{array}{c}\text { Decreto } \mathrm{n}^{\circ} 88.218, \text { de } 06 \text { de } \\
\text { abril de } 1983\end{array}$ & $87.943,14$ & Caravelas, Alcobaça - BA & Marinho & Sim \\
\hline $\begin{array}{l}\text { REBIO DE } \\
\text { COMBOIOS }\end{array}$ & $\begin{array}{c}\text { Decreto } n^{\circ} 90.222, \text { de } 25 \text { de } \\
\text { setembro de } 1984\end{array}$ & 784,63 & Linhares, Aracruz - ES & Costeiro & Em const. \\
\hline ESEC DO TAIM & $\begin{array}{c}\text { Decreto } n^{\circ} 92.963, \text { de } 21 \text { de } \\
\text { julho de } 1986\end{array}$ & $32.806,31$ & $\begin{array}{c}\text { Santa Vitória do Palmar, Rio } \\
\text { Grande - RS }\end{array}$ & $\begin{array}{l}\text { Marinho } \\
\text { Costeiro }\end{array}$ & Sim \\
\hline $\begin{array}{l}\text { ESEC DOS } \\
\text { TUPINIQUINS }\end{array}$ & $\begin{array}{c}\text { Decreto } n^{\circ} 92.964, \text { de } 21 \text { de } \\
\text { julho de } 1986\end{array}$ & $1.727,70$ & $\begin{array}{l}\text { Peruíbe, Cananéia, } \\
\text { Itanhaém - SP }\end{array}$ & Marinho & Sim \\
\hline $\begin{array}{l}\text { PARNA DA LAGOA } \\
\text { DO PEIXE }\end{array}$ & $\begin{array}{c}\text { Decreto } n^{\circ} 93.546, \text { de } 06 \text { de } \\
\text { novembro de } 1986\end{array}$ & $36.721,71$ & $\begin{array}{c}\text { Mostardas, Tavares, São } \\
\text { José do Norte - RS }\end{array}$ & $\begin{array}{l}\text { Marinho } \\
\text { Costeiro }\end{array}$ & $\begin{array}{l}\text { Em } \\
\text { constr. }\end{array}$ \\
\hline ESEC DE CARIJÓS & $\begin{array}{l}\text { Decreto } n^{\circ} 94.656, \text { de } 20 \text { de } \\
\text { julho de } 1987\end{array}$ & 759,33 & Florianópolis - SC & $\begin{array}{l}\text { Marinho } \\
\text { Costeiro }\end{array}$ & Sim \\
\hline ESEC TUPINAMBÁS & $\begin{array}{c}\text { Decreto } n^{\circ} 94.656, \text { de } 20 \text { de } \\
\text { julho de } 1987\end{array}$ & $2.463,59$ & $\begin{array}{c}\text { Praia Grande, São } \\
\text { Sebastião, Ubatuba - SP }\end{array}$ & Marinho & $\begin{array}{c}\text { Em } \\
\text { constr. }\end{array}$ \\
\hline $\begin{array}{l}\text { PARNA MARINHO } \\
\text { DE FERNANDO DE } \\
\text { NORONHA }\end{array}$ & $\begin{array}{l}\text { Decreto } n^{\circ} 96.693, \text { de } 14 \text { de } \\
\text { setembro de } 1988\end{array}$ & $10.929,47$ & $\begin{array}{c}\text { Fernando de Noronha } \\
\text { (Distrito Estadual) - PE } \\
\text { Caravelas - BA }\end{array}$ & Marinho & Sim \\
\hline $\begin{array}{l}\text { REBIO DE SANTA } \\
\text { ISABEL }\end{array}$ & $\begin{array}{l}\text { Decreto } n^{\circ} 96.999, \text { de } 20 \text { de } \\
\text { outubro de } 1988\end{array}$ & $4.109,88$ & Pirambu, Pacatuba - SE & Costeiro & Não \\
\hline $\begin{array}{l}\text { PARNADO } \\
\text { SUPERAGUI }\end{array}$ & $\begin{array}{l}\text { Decreto } n^{\circ} 97.688 \text {, de } 25 \text { de } \\
\text { abril de } 1989\end{array}$ & $33.860,36$ & Guaraqueçaba - PR & Costeiro & Não \\
\hline ESEC DE TAMOIOS & $\begin{array}{c}\text { Decreto } n^{\circ} 98.864 \text {, de } 23 \text { de } \\
\text { janeiro de } 1990\end{array}$ & $8.660,35$ & Parati, Angra dos Reis - RJ & Marinho & Sim \\
\hline $\begin{array}{l}\text { REBIO MARINHA DO } \\
\text { ARVOREDO }\end{array}$ & $\begin{array}{l}\text { Decreto } \mathrm{n}^{\circ} 99.142, \text { de } 12 \text { de } \\
\text { março de } 1990\end{array}$ & $17.104,60$ & $\begin{array}{c}\text { Governador Celso Ramos, } \\
\text { Bombinhas - SC }\end{array}$ & Marinho & Sim \\
\hline $\begin{array}{l}\text { PARNA RESTINGA } \\
\text { DE JURUBATIBA }\end{array}$ & $\begin{array}{c}\text { Decreto } s / n^{\circ}, \text { de } 29 \text { de abril de } \\
1998\end{array}$ & $14.919,46$ & $\begin{array}{c}\text { Carapebus, Quissamã, } \\
\text { Macaé - RJ }\end{array}$ & Costeiro & Sim \\
\hline $\begin{array}{l}\text { PARNA DE SAINT- } \\
\text { HILAIRE/LANGE }\end{array}$ & $\begin{array}{c}\text { Lei } n^{\circ} 10.227 \text {, de } 23 \text { de maio } \\
\text { de } 2001\end{array}$ & $24.352,43$ & $\begin{array}{l}\text { Guaratuba, Paranaguá, } \\
\text { Matinhos, Morretes - PR }\end{array}$ & Costeiro & Sim \\
\hline
\end{tabular}




\begin{tabular}{|c|c|c|c|c|c|}
\hline $\begin{array}{l}\text { Unidades de } \\
\text { conservação }\end{array}$ & Ato legal & Área (ha) & Municípios & Ecossistema & $\begin{array}{c}\text { Plano de } \\
\text { manejo }\end{array}$ \\
\hline $\begin{array}{l}\text { PARNADE } \\
\text { JERICOACOARA }\end{array}$ & $\begin{array}{l}\text { Decreto } s / n^{\circ} \text {, de } 04 \text { de } \\
\text { fevereiro de } 2002\end{array}$ & $8.863,03$ & $\begin{array}{c}\text { Jijoca de Jericoacoara, Cruz } \\
\text { - CE }\end{array}$ & $\begin{array}{l}\text { Marinho } \\
\text { Costeiro }\end{array}$ & Sim \\
\hline $\begin{array}{l}\text { REVIS ILHA DOS } \\
\text { LOBOS }\end{array}$ & $\begin{array}{c}\text { Decreto } s / n^{0} \text {, de } 04 \text { de julho de } \\
2005\end{array}$ & 142,39 & Torres, Porto Alegre - RS & Marinho & Sim \\
\hline $\begin{array}{c}\text { ESEC DA } \\
\text { GUANABARA }\end{array}$ & $\begin{array}{l}\text { Decreto } s / n^{\circ} \text {, de } 15 \text { de } \\
\text { fevereiro de } 2006\end{array}$ & $1.936,23$ & $\begin{array}{l}\text { Guapimirim, Itaboraí, São } \\
\text { Gonçalo - RJ }\end{array}$ & $\begin{array}{l}\text { Marinho } \\
\text { Costeiro }\end{array}$ & Sim \\
\hline $\begin{array}{l}\text { REVIS DO RIO DOS } \\
\text { FRADES* }\end{array}$ & $\begin{array}{l}\text { Decreto } s / n^{\circ} \text {, de } 21 \text { de } \\
\text { dezembro de } 2007\end{array}$ & 898,67 & Porto Seguro - BA & Costeiro & Não \\
\hline REVIS DE UNA & $\begin{array}{l}\text { Decreto } s / n^{\circ} \text {, de } 21 \text { de } \\
\text { dezembro de } 2007\end{array}$ & $23.262,09$ & Una - BA & Costeiro & Sim \\
\hline $\begin{array}{l}\text { MONA DAS ILHAS } \\
\text { CAGARRAS }\end{array}$ & $\begin{array}{l}\text { Lei } n^{\circ} 12.229 \text {, de } 13 \text { de abril de } \\
2010\end{array}$ & 105,93 & Rio de Janeiro, Niterói - RJ & Marinho & Não \\
\hline $\begin{array}{l}\text { REVIS DE SANTA } \\
\text { CRUZ }\end{array}$ & $\begin{array}{c}\text { Decreto } s / n^{\circ} \text {, de } 17 \text { de junho de } \\
2010\end{array}$ & $17.709,39$ & Aracruz - ES & Marinho & Não \\
\hline $\begin{array}{l}\text { PARNA MARINHO } \\
\text { DAS ILHAS DOS } \\
\text { CURRAIS }\end{array}$ & $\begin{array}{c}\text { Lei } n^{\circ} 12.829, \text { de } 20 \text { de junho } \\
\text { de } 2013\end{array}$ & $1.359,70$ & Guaratuba, Paranaguá - PR & Marinho & Não \\
\hline $\begin{array}{c}\text { REVIS DO } \\
\text { ARQUIPÉLAGO DE } \\
\text { ALCATRAZES }\end{array}$ & $\begin{array}{c}\text { Decreto } s / n^{\circ}, \text { de } 02 \text { de agosto } \\
\text { de } 2016\end{array}$ & $67.479,29$ & São Sebastião - SP & Marinho & $\begin{array}{c}\text { Em } \\
\text { constr. }\end{array}$ \\
\hline $\begin{array}{l}\text { MONA DO } \\
\text { ARQUIPÉLAGO DE } \\
\text { SÃO PEDRO E SÃO } \\
\text { PAULO }\end{array}$ & $\begin{array}{l}\text { Decreto } \mathrm{n}^{\circ} 9.313, \text { de } 19 \text { de } \\
\text { março de } 2018\end{array}$ & $4.726 .317,84$ & Pernambuco & Marinho & Não \\
\hline $\begin{array}{l}\text { MONA DAS ILHAS } \\
\text { DE TRINDADE, } \\
\text { MARTIM VAZ E DO } \\
\text { MONTE COLUMBIA }\end{array}$ & $\begin{array}{l}\text { Decreto } \mathrm{n}^{\circ} 9.312 \text {, de } 19 \text { de } \\
\text { março de } 2018\end{array}$ & $6.769 .671,75$ & Vitória - ES & Marinho & Não \\
\hline \multicolumn{6}{|c|}{$\begin{array}{l}\text { *Correspondem a UCs do Bioma Mata Atlântica, segundo ICMBio (2018). } \\
\text { Fonte: ICMBio (2018), ISA (2-18), MMA (2018), WWF (2018). }\end{array}$} \\
\hline $\begin{array}{c}\text { Unidades de } \\
\text { Conservação } \\
\end{array}$ & Ato Legal & Área (ha) & Município & Ecossistema & $\begin{array}{c}\text { Plano de } \\
\text { Manejo } \\
\end{array}$ \\
\hline APADE PIAÇABUÇÚ & $\begin{array}{c}\text { Decreto } n^{\circ} 88.421, \text { de } 21 \text { de } \\
\text { junho de } 1983\end{array}$ & $9.107,01$ & $\begin{array}{c}\text { Feliz Deserto, Piaçabuçu - } \\
\text { AL }\end{array}$ & Costeiro & Sim \\
\hline APADO CAIRUÇU & $\begin{array}{l}\text { Decreto } n^{\circ} 89.242 \text {, de } 27 \text { de } \\
\text { dezembro de } 1983\end{array}$ & $32.610,78$ & Parati - RJ & $\begin{array}{l}\text { Marinho } \\
\text { Costeiro }\end{array}$ & Sim \\
\hline $\begin{array}{l}\text { APADE GUAPI- } \\
\text { MIRIM }\end{array}$ & $\begin{array}{l}\text { Decreto } n^{\circ} 90.225, \text { de } 25 \text { de } \\
\text { setembro de } 1984\end{array}$ & $13.890,54$ & $\begin{array}{l}\text { Guapimirim, Itaboraí, Magé, } \\
\text { São Gonçalo - RJ }\end{array}$ & $\begin{array}{l}\text { Marinho } \\
\text { Costeiro }\end{array}$ & Sim \\
\hline $\begin{array}{l}\text { APADE CANANÉIA- } \\
\text { IGUAPE-PERUÍBE }\end{array}$ & $\begin{array}{c}\text { Decreto } n^{\circ} 90.347, \text { de } 23 \text { de } \\
\text { outubro de } 1984\end{array}$ & $202.309,58$ & $\begin{array}{c}\text { Iguape, Cananéia, llha } \\
\text { Comprida, Peruíbe, Itariri - } \\
\text { SP }\end{array}$ & Costeiro & Sim \\
\hline $\begin{array}{c}\text { APA } \\
\text { GUARAQUEÇABA }\end{array}$ & $\begin{array}{c}\text { Decreto } n^{\circ} 90.883, \text { de } 31 \text { de } \\
\text { janeiro de } 1985\end{array}$ & $282.446,36$ & $\begin{array}{c}\text { Guaraqueçaba, Antonina, } \\
\text { Paranaguá, Campina } \\
\text { Grande do Sul - PR }\end{array}$ & $\begin{array}{l}\text { Marinho } \\
\text { Costeiro }\end{array}$ & Sim \\
\hline $\begin{array}{l}\text { ARIE ILHA DO } \\
\text { AMEIXAL } \\
\text { ARIE ILHAS DA }\end{array}$ & $\begin{array}{c}\text { Decreto } n^{\circ} 91.889 \text { de } 05 \text { de } \\
\text { novembro de } 1985\end{array}$ & 358,88 & Peruibe & Costeiro & Sim \\
\hline $\begin{array}{l}\text { QUEIMADA } \\
\text { PEQUENA E } \\
\text { QUEIMADA }\end{array}$ & $\begin{array}{c}\text { Decreto } \mathrm{n}^{\circ} 91.887, \text { de } 05 \text { de } \\
\text { novembro de } 1985\end{array}$ & 65,17 & Peruibe - SP & Marinho & Não \\
\hline $\begin{array}{l}\text { GRANDE } \\
\text { APADE FERNANDO }\end{array}$ & & & & & \\
\hline $\begin{array}{l}\text { DE NORONHA - } \\
\text { ROCAS - SÃO } \\
\text { PEDRO E SÃO } \\
\text { PAULO }\end{array}$ & $\begin{array}{c}\text { Decreto } n^{\circ} 92.755 \text { de } 05 \text { de } \\
\text { junho de } 1986\end{array}$ & $154.409,03$ & $\begin{array}{c}\text { Fernando de Noronha } \\
\text { (Distrito Estadual) - PE } \\
\text { Caravelas - BA }\end{array}$ & Marinho & Sim \\
\hline
\end{tabular}




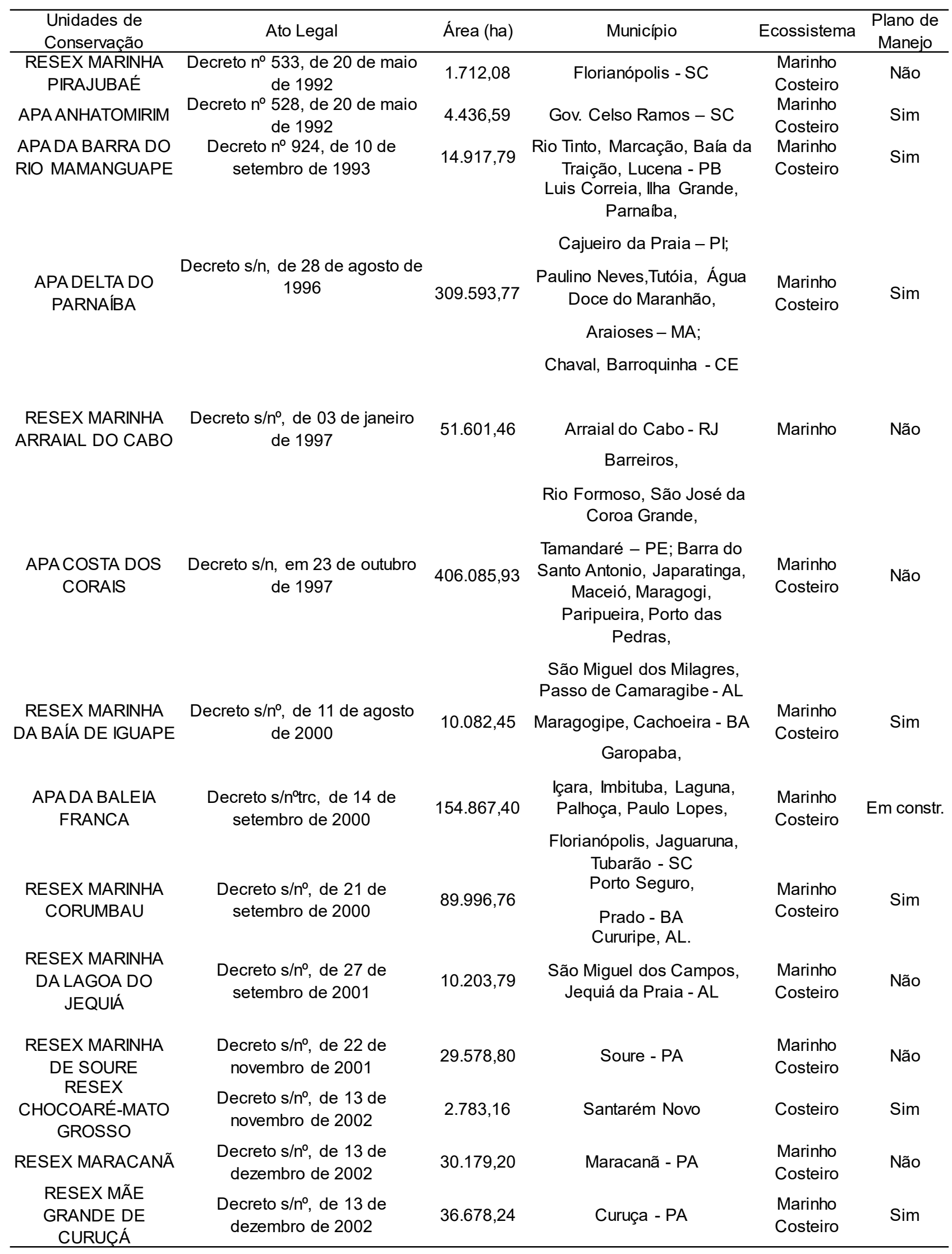




\begin{tabular}{|c|c|c|c|c|c|}
\hline $\begin{array}{l}\text { Unidades de } \\
\text { Conservação }\end{array}$ & Ato Legal & Área (ha) & Município & Ecossistema & $\begin{array}{l}\text { Plano de } \\
\text { Manejo }\end{array}$ \\
\hline $\begin{array}{l}\text { RESEX MÂE } \\
\text { GRANDE DE } \\
\text { CURUÇÁ }\end{array}$ & $\begin{array}{l}\text { Decreto } s / n^{\circ} \text {, de } 13 \text { de } \\
\text { dezembro de } 2002\end{array}$ & $36.678,24$ & Curuça - PA & $\begin{array}{l}\text { Marinho } \\
\text { Costeiro }\end{array}$ & Sim \\
\hline $\begin{array}{l}\text { RESEX DE SÃO } \\
\text { JOÃO DA PONTA }\end{array}$ & $\begin{array}{l}\text { Decreto } s / n^{0} \text {, de } 13 \text { de } \\
\text { dezembro de } 2002\end{array}$ & $3.409,44$ & São João da Ponta - PA & Costeiro & Sim \\
\hline RESEX BATOQUE & $\begin{array}{c}\text { Decreto } s / n^{\circ} \text {, de } 05 \text { de junho de } \\
2003\end{array}$ & 601,44 & Aquiraz - CE & $\begin{array}{l}\text { Marinho } \\
\text { Costeiro }\end{array}$ & Sim \\
\hline $\begin{array}{l}\text { RESEX DE } \\
\text { CURURUPU }\end{array}$ & $\begin{array}{c}\text { Decreto } s / n^{\circ}, \text { de } 02 \text { de junho de } \\
2004\end{array}$ & $186.053,87$ & $\begin{array}{l}\text { Serrano do Maranhão, } \\
\text { Cururupu - MA; }\end{array}$ & $\begin{array}{l}\text { Marinho } \\
\text { Costeiro }\end{array}$ & Sim \\
\hline $\begin{array}{l}\text { RESEX MARINHA } \\
\text { DE GURUPI-PIRIÁ }\end{array}$ & $\begin{array}{l}\text { Decreto } s / n^{\circ} \text {, de } 20 \text { de maio de } \\
2005\end{array}$ & $74.081,81$ & Viseu - PA & $\begin{array}{l}\text { Marinho } \\
\text { Costeiro }\end{array}$ & Não \\
\hline $\begin{array}{l}\text { RESEX ARAÍ- } \\
\text { PEROBA }\end{array}$ & $\begin{array}{c}\text { Decreto } s / n^{\circ}, \text { de } 20 \text { de maio de } \\
2005\end{array}$ & $11.549,73$ & Augusto Corrêa -PA & $\begin{array}{l}\text { Marinho } \\
\text { Costeiro }\end{array}$ & Não \\
\hline $\begin{array}{l}\text { RESEX MARINHA } \\
\text { DE CAETÉ- } \\
\text { TAPERAÇU }\end{array}$ & $\begin{array}{l}\text { Decreto } s / n^{\circ} \text {, de } 20 \text { de maio de } \\
2005\end{array}$ & $42.489,17$ & Bragança - PA & $\begin{array}{l}\text { Marinho } \\
\text { Costeiro }\end{array}$ & Sim \\
\hline $\begin{array}{l}\text { RESEX MARINHA } \\
\text { DE TRACUATEUA }\end{array}$ & $\begin{array}{c}\text { Decreto } s / n^{\circ} \text {, de } 20 \text { de maio de } \\
2005\end{array}$ & $27.864,08$ & Tracuateua, Bragança - PA & $\begin{array}{l}\text { Marinho } \\
\text { Costeiro }\end{array}$ & Não \\
\hline $\begin{array}{l}\text { RESEX } \\
\text { CANAVIEIRAS }\end{array}$ & $\begin{array}{c}\text { Decreto } s / n^{\circ}, \text { de } 05 \text { de junho de } \\
2006\end{array}$ & $100.726,36$ & Canavieiras, Belmonte - BA & $\begin{array}{l}\text { Marinho } \\
\text { Costeiro }\end{array}$ & Sim \\
\hline $\begin{array}{l}\text { RESEX ACAÚ- } \\
\text { GOIANA }\end{array}$ & $\begin{array}{c}\text { Decreto } s / n^{\circ}, \text { de } 26 \text { de } \\
\text { setembro de } 2007\end{array}$ & $6.676,69$ & $\begin{array}{c}\text { Goiana, } \\
\text { Pitimbu, Caaporã - PB }\end{array}$ & $\begin{array}{l}\text { Marinho } \\
\text { Costeiro }\end{array}$ & Sim \\
\hline $\begin{array}{l}\text { RESEX PRAINHA } \\
\text { DO CANTO VERDE }\end{array}$ & $\begin{array}{c}\text { Decreto } s / n^{\circ}, \text { de } 05 \text { de junho de } \\
2009\end{array}$ & $29.804,99$ & Beberibe - CE & $\begin{array}{l}\text { Marinho } \\
\text { Costeiro }\end{array}$ & Não \\
\hline $\begin{array}{l}\text { RESEX DE } \\
\text { CASSURUBÁ }\end{array}$ & $\begin{array}{l}\text { Decreto } s / n^{\circ} \text {, de } 05 \text { de junho de } \\
2009\end{array}$ & $100.767,56$ & $\begin{array}{c}\text { Alcoçaba, Caravelas, Nova } \\
\text { Viçosa-BA }\end{array}$ & $\begin{array}{l}\text { Marinho } \\
\text { Costeiro }\end{array}$ & Sim \\
\hline $\begin{array}{l}\text { APACOSTA DAS } \\
\text { ALGAS }\end{array}$ & $\begin{array}{l}\text { Decreto } s / n^{\circ} \text {, de } 17 \text { de junho de } \\
2010\end{array}$ & $115.001,92$ & $\begin{array}{c}\text { Aracruz, } \\
\text { Fundão, Serra - ES. }\end{array}$ & $\begin{array}{l}\text { Marinho } \\
\text { Costeiro }\end{array}$ & Não \\
\hline $\begin{array}{l}\text { RESEX } \\
\text { MARINHA MESTRE } \\
\text { LUCINDO }\end{array}$ & $\begin{array}{c}\text { Decreto } s / n^{\circ} \text {, de } 10 \text { de outubro } \\
\text { de } 2014\end{array}$ & $26.464,88$ & Marapanim - PA & $\begin{array}{l}\text { Marinho } \\
\text { Costeiro }\end{array}$ & Sim \\
\hline $\begin{array}{l}\text { RESEX MARINHA } \\
\text { MOCAPAJUBA** }\end{array}$ & $\begin{array}{c}\text { Decreto } s / n^{\circ}, 10 \text { de outubro de } \\
2014\end{array}$ & $21.027,80$ & $\begin{array}{c}\text { São Caetano de Odivelas - } \\
\text { PA }\end{array}$ & $\begin{array}{l}\text { Marinho } \\
\text { Costeiro }\end{array}$ & Sim \\
\hline $\begin{array}{c}\text { APAARQUIPÉLAGO } \\
\text { DE TRINDADE E } \\
\text { MARTIM VAZ } \\
\text { APADO }\end{array}$ & $\begin{array}{c}\text { Decreto } n^{\circ} 9.312, \text { de } 19 \text { de } \\
\text { março de } 2018\end{array}$ & $40.385 .419,6$ & Vitória - ES & Marinho & Não \\
\hline $\begin{array}{l}\text { ARQUIPÉLAGO DE } \\
\text { SÃO PEDRO E SÃO } \\
\text { PAULO }\end{array}$ & $\begin{array}{c}\text { Decreto } n^{\circ} 9.313 \text {, de } 19 \text { de } \\
\text { março de } 2018\end{array}$ & $38.450 .193,8$ & Pernambuco & Marinho & Não \\
\hline $\begin{array}{c}\text { RESEX } \\
\text { TAPETININGA }\end{array}$ & $\begin{array}{c}\text { Decreto } n^{\circ} 9.333 \text {, de } 5 \text { de abril } \\
\text { de } 2018\end{array}$ & $16.294,64$ & Bequimão - MA & Costeiro & Não \\
\hline $\begin{array}{c}\text { RESEX } \\
\text { ARAPIRANGA- } \\
\text { TROMAÍ }\end{array}$ & $\begin{array}{c}\text { Decreto } n^{\circ} 9.339 \text {, de } 5 \text { abril de } \\
2018\end{array}$ & $186.909,14$ & Luís Domingues - MA & $\begin{array}{l}\text { Marinho } \\
\text { Costeiro }\end{array}$ & Não \\
\hline $\begin{array}{l}\text { RESEX DABAÍA DO } \\
\text { TUBARÃO }\end{array}$ & $\begin{array}{l}\text { Decreto } n^{\circ} 9.340, \text { de } 5 \text { de abril } \\
\text { de } 2018\end{array}$ & $223.888,98$ & $\begin{array}{l}\text { Humberto de Campos, } \\
\text { Icatu - MA }\end{array}$ & $\begin{array}{l}\text { Marinho } \\
\text { Costeiro } \\
\end{array}$ & Não \\
\hline
\end{tabular}

*Correspondem a UCs do Bioma Mata Atlântica, segundo ICMBio (2018).

Fonte: ICMBio (2018), ISA (2-18), MMA (2018), WWF (2018).

Das 41 UCs de uso sustentável levantadas, quatro $(10 \%)$ são exclusivamente marinhas incluindo as ilhas, seis (15\%) são costeiras e 30 (75\%) marinha-costeiras. Na Figura 2 é possível observar a situação quanto ao tamanho e quantidade de UCs ao longo das décadas. 


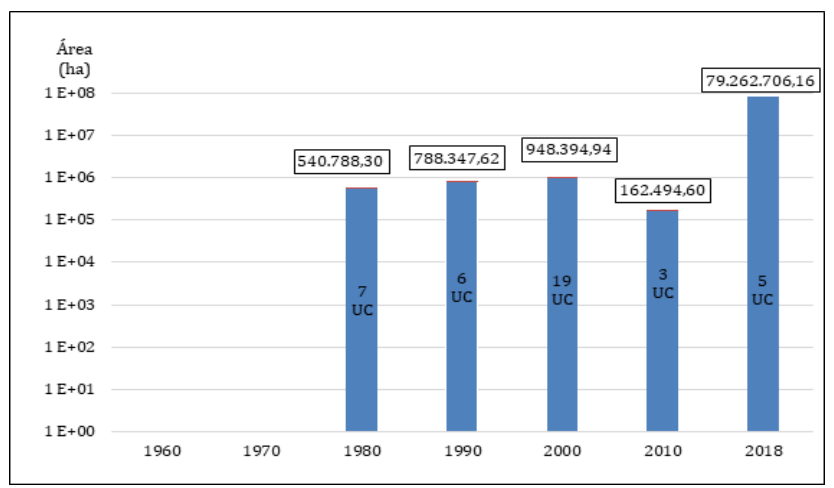

Figura 2 - Representação das Unidades de Conservação de uso sustentável marinhas e/o costeiras em relação a sua área e quantidade ao longo das décadas.

\section{DISCUSSÃO}

A primeira UC de proteção integral criada em ecossistema costeiro foi o Parque Nacional de Monte Pascoal, em 1961, e em ecossistema marinho foi a Reserva Biológica de Atol das Rocas, em 1979. Portanto, somente 24 e 42 anos, respectivamente, após a criação da primeira unidade de conservação terrestre brasileira, PARNA Itatiaia, denotando uma resposta lenta comparada ao processo mundial de medidas de proteção ambiental de ecossistemas marinhos e costeiros. Na década de 1920, o aumento do interesse pelas ciências marinhas levou ao estabelecimento de pequenas reservas de pesquisa científica, como o Laboratório Friday Harbor (Washington) que designou uma Reserva Biológica Marinha, em 1923 (National Academy of Sciences, 2001). Quanto às UCs de uso sustentável, as primeiras contendo ecossistemas costeiros foram as APAs de Piaçabuçu e do Cairuçu, ambas criadas em 1993. Exclusivamente em ecossistema marinho tem-se a ARIE Ilhas da Queimada Pequena e Queimada Grande, criada em 1985. Portanto, respectivamente, onze e três anos, respectivamente, após a criação das primeiras UCs de uso sustentável terrestres, as APAs da Região Serrana de Petrópolis e Bacia do Rio Paraíba do Sul, ambas criadas em setembro de 1982, às quais também tardaram a ocorrer se comparadas ao grupo de proteção integral.

O Brasil possui 334 unidades de conservação federais (ICMBio, 2019), das quais 149 são de proteção integral (44\%). Rylands \& Brandon (2005) constataram que em 2005 apenas na Amazônia existia um equilíbrio aproximado entre UCs de proteção integral (49\%) e uso sustentável (51\%), sendo que para o Bioma Marinho havia um desiquilíbrio com $74 \%$ de UCs de uso sustentável. Atualmente, segundo nosso levantamento, esta discrepância diminuiu, com $45 \%$ de UCs que compõe grupo de proteção integral. As AMPs pertencentes ao grupo de proteção integral tendem a ser mais eficientes na conservação da biodiversidade, considerando os seus objetivos mais restritivos, mas dada a possibilidade da pesca artesanal em algumas UCs de uso sustentável e a importância dessa atividade como fonte socioeconômica, podem seruma opção viável desde que exista gestão eficiente (Steiner, 2015).

Pode-se observar um crescimento considerável de UCs de proteção integral na década de $80 \mathrm{com}$ a criação de 15 unidades com área ultrapassando um milhão de hectares, enquanto que na década de 60 existia apenas uma contendo ecossistema costeiro e na década de 70 , duas, sendo uma de ecossistema costeiro e outra de marinho. A explosão de UCs na década de 80 somente foi superada no ano de 2018 com a criação das duas UCs Marinhas, os Monumentos Naturais, Arquipélago de São Pedro e São Paulo e Trindade Martim Vaz, as duas representando mais de 11 milhões de hectares. Também para as UCs de uso sustentável nota-se ampliação em número e tamanho, especialmente em 2018, representado apenas com a criação de duas novas UCs, a APA Arquipélago de São Pedro e São Paulo e a APA Arquipélago de Trindade Martim e Vaz, 99,4\% de área das cinco UCs criadas nesse ano.

Cabe destacar que a situação de UCs marinhas e/ou costeiras no Brasil refletem melhorias, pois eram insuficientes em número e extensão (Amaral \& Jablonski, 2005) e especialmente com pouca representação de ecossistema marinho (Prates \& Pereira, 2000). Contudo, paraArtaza-Barrios \& Schiavetti (2007) fica evidente que apenas o ato administrativo de criar uma UC não garante sua efetiva implementação, pois requerendo do órgão gestor (governo federal, estadual, municipal ou proprietário particular) estabelecer as condições adequadas de infraestrutura, de pessoal e de regularização fundiária que permitam de fato proteger a área.

Até 2017 as UCs de proteção integral de maior dimensão se referiam ao Parque Nacional do Cabo Orange, Marinho Costeiro, com 657.318,06 ha, seguido do Parque Nacional Marinho dos Abrolhos, com $87.943,14$ ha. Contudo, em 2018 a representatividade em área de UCs marinhas se elevou com a decretação do Monumento Natural das Ilhas de Trindade, Martim Vaz e do Monte Columbia, com 6.915.536,11 ha e do Monumento Natural Arquipélago de São Pedro e São Paulo, 4.726.317,84 ha. Esses resultados atendem a Meta de 11 de Aichi e os de ODS 14, que preveem a proteção de $17 \%$ das áreas marinhas e costeiras de cada país até 2020. O Brasil passou de 1,5\% de áreas marinhas protegidas para $25 \%$, e com a inclusão destes arquipélagos, todas as ilhas oceânicas brasileiras, que incluem também as ilhas de Fernando de Noronha e oAtol das Rocas, passam a ser protegidas por unidades de conservação (ICMBio, 2018).

Do total de UCs 43 (48\%) possuem Plano de Manejo e cinco estão em construção. Muitas unidades 
de conservação não tiveram seus planos de manejo elaborados ou implementados ou carecem de infraestrutura para efetivá-las (Amaral e Jablonski, 2005). Das 34 UCs estudadas em Minas Gerais, 87\% não possuíam Plano de Manejo, sendo essa a realidade de outros estados, como São Paulo, Rio de Janeiro e Mato Grosso (Lima et al., 2005). Além da quantidade de UCs sem Plano de Manejo, é preocupante verificar que a REBIO do Lago Piratuba foi criada em 1980 e ainda não possui este instrumento de gestão, assim como o caso da ARIE Ilhas da Queimada Pequena e Queimada Grande, que desde a sua criação completa 34 anos sem Plano de Manejo ParaArtaza-Barrios \& Schiavetti (2007), a elaboração de bons planos de manejo, adequados à realidade da UC é uma importante ferramenta para a implementação das mesmas.

\section{CONCLUSÃO}

As unidades de conservação marinhas e costeiras passaram a ser criadas apenas a partir da décadade60, evoluindoaolongo dotempoemquantidade e de representatividade de área. Contudo, muitas delas ainda não têm Plano de Manejo, fato que denota deficiência no esforço de efetividade na proteção.

\section{REFERÊNCIAS}

Amaral, A. C.\& Jablonski, S. 2005. Conservação da biodiversidade marinha e costeira no Brasil. Megadiversidade, Belo Horizonte. 1(1): 200-208.

Artaza-Barrios, O. H. \& Schiavetti, A. 2007. Análise da Efetividade do Manejo de duas Áreas de Proteção Ambiental do Litoral Sul da Bahia, Brasil. Revista da Gestão Costeira Integrada 7(2): 117-128.

Drummond, J.; Franco, J. L. A. \& Oliveira, D. 2000. Uma análise sobre a história e a situação das unidades de conservação no Brasil In: Ganem, R. S. Conservação da biodiversidade: legislação e políticas públicas. Brasília: Câmara dos Deputados, Edições Câmara. 341-385.

ICMBio - Instituto Chico Mendes de Conservação da Biodiversidade. Efetividade da Gestão de UCs. Disponível em: <http://www.icmbio.gov.br/portal/ efetividadedagestaodeucs>. Acesso em: 2018

ICMBio. Unidades de Conservação. Disponível em: <http://www.icmbio.gov.br/portal/ unidadesdeconservacao/biomas-brasileiros>. Acesso em: 03 set. 2019.

IUCN. 2008. Guidelines for ApplyingProtectedArea Management Categories. Gland: IUCN/WCPA/ FundaciónBiodiversidad/Junta de Andalucia.

Lima, G. S.; Ribeiro, G. A. \& Gonçalves. W. 2005. Avaliação da efetividade de manejo das unidades de conservação de proteção integral em Minas Gerais. Árvore, Viçosa-MG, 29 (4): 647-653.

Marenzi, R. C.; Voigt, N. \& Polette, M. 2013. Parques como áreas legalmente protegidas e estratégias à sustentabilidade. In: Maria Cláudia da Silva Antunes de Souza; Denise Schmitt Siqueira Garcia. (Org.). Direito Ambiental, Transnacionalidade e Sustentabilidade. 1ed. Itajaí: UNIVALI. 1: 201-221.

Martins, L.; Marenzi, R.C. \& Lima,A. 2015. Levantamento e representatividade das unidades de conservação instituídas no estado de Santa Catarina, Brasil. Desenvolv. Meio Ambiente. 33: 241-259.

Medeiros, Rodrigo. 2006. Evolução das tipologias e categorias de áreas protegidas no Brasil. Ambiente \& Sociedade. 9 (1): 41-64.

MMA-Ministério do MeioAmbiente. CadastrodeUnidades de Conservação: Disponível em: <www.mma.gov. br/cadastro_uc>. Acesso em: 03 set. 2019.

National Academy of Sciences. 2001. Historical Background and Evaluationof Marine Protected Areas in the United States In Marine Protected Areas: Tools for Sustaining Ocean Ecosystems. Washington DC: National Academy of Sciences. 145-174.

Prates, A.P.L. \& Pereira, P.M. 2000. Representatividade das Unidades de Conservação Costeiro-marinhas: Análise e sugestões. Anais Congresso Brasileiro de Unidades de Conservação, Campo Grande. 3: 784-793.

Rylands, A.B. \& Brandon, K. 2005. Unidades de Conservação brasileiras. Megadiversidade, 1(1): 27-35.

Salm, R. V.; Clark, J. \& Siirila, E. 2000. Marine and Coastal Protected Areas: A Guide for Planners and Managers. 3 ed. Washington: IUCN.

Soares, M. O.; Paiva, C. C.; Freitas, J. E. P. \& Lotufo, T. M. 2011. Gestão de unidades de conservação marinhas: o caso do Parque Estadual Marinho da Pedra da Risca do Meio, NE - Brasil. Revista da Gestão Costeira Integrada 11(2):257-268.

Steiner, L. 2015. Avaliação da efetividade das Áreas Marinhas Protegidas: um estudo de caso no estado de Santa Catarina. Trabalho de Conclusão de Curso (Oceanografia) - Centro de Ciências Tecnológicas da Terra e do Mar, Universidade do Vale do Itajaí, Itajaí.

Terborgh, J.; C. Van Schaik; L. Davenport \& M. Rao, 2002. Tornando os parques eficientes: estratégias para conservação da natureza nos trópicos. Curitiba. Editora da UFPR, Fundação O Boticário de Proteção à Natureza.

Submetido: Dezembro/19 Revisado: Julho/20 Aceito:Julho/20 Publicado: 06 de Fevereiro/2021 